\title{
Sensors in Network (5) -Future Sensor Systems in Internet of Things or Trillion Sensor Universe-
}

\author{
Kazusuke Maenaka* \\ Department of Electronics and Computer Science, Graduate School of Engineering, University of Hyogo, \\ 2167 Shosha, Himeji, Hyogo 671-2280, Japan
}

(Received October 17, 2016; accepted November 8, 2016)

Keywords: IoT, Trillion Sensor Universe, big data, actuator

\section{Introduction}

In this perspective series, I wrote four reports under the theme of Sensors in Network. Through these reports, I explained that sensors with simple sensing functions alone had made progress, which led to the realization of higher functions of sensors. In addition, I introduced MEMS technologies that realized this advancement, methods for integrating multiple types of sensor in a package and connecting them to a network, and the human activity monitoring system as an example of typical systems. This is the final session of this perspective series. Here, I will discuss my vision toward the future sensor networks and the required technologies to achieve them.

\section{Internet of Things (IoT) and Trillion Sensor Universe}

IoT was proposed by Kevin Ashton. ${ }^{(1)}$ This is a concept for improving the entire society, such as by reducing various costs and increasing safety and convenience. In the IoT society, not only computers but also various "things" are connected to the Internet, enabling data to be analyzed and highly controlled by exchanging and sharing information. With this concept, sensors that serve as "things" for collecting data play an extremely important role. Cisco Systems, a manufacturer of network equipment, predicted that the number of devices connected to the network was one per person in the world already in 2008 and it would exceed 50 billion (sevenfold greater than the total world population) in 2020 (Fig. 1). ${ }^{(2)}$ In line with this wide spread of devices, the annual number of sensors used is expected to exceed a trillion in the 2020s (Fig. 2). (3) The above are the prediction of the advancement of IoT and Trillion Sensor Universe. Although you may consider this prediction to be excessive, it is not so unrealistic when taking into account the speed of previous advancements in sensor systems and RF technologies. As shown in the previous sessions, ${ }^{(4-7)}$ connecting sensor systems to the Internet has been yearly becoming easy owing to the development of highly advanced sensors, cost reduction, realization of combo sensors, incorporation of RF functions into sensors, and reduction in power consumption of hardware. For example, it is already possible to incorporate RF sensor nodes into commonly used shoes (for monitoring a walking person), bags (for preventing their misplacement and confirming their position), and clothes (for monitoring human

*Corresponding author: e-mail: maenaka@eng.u-hyogo.ac.jp 


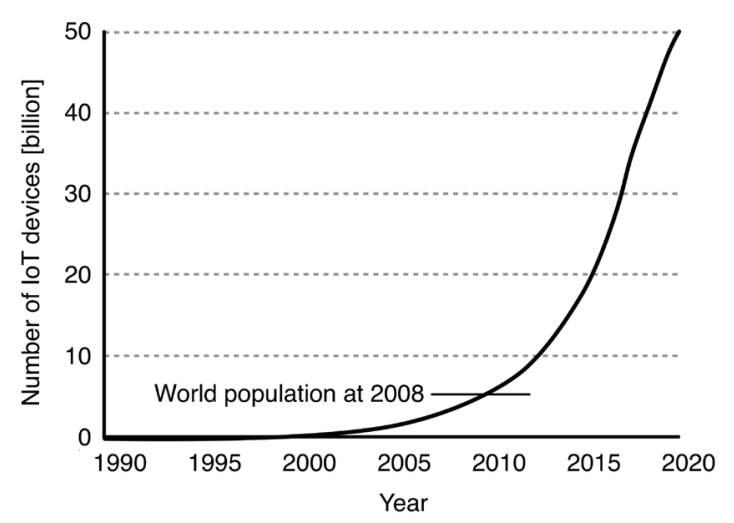

Fig. 1. Prediction of growth of IoT devices.

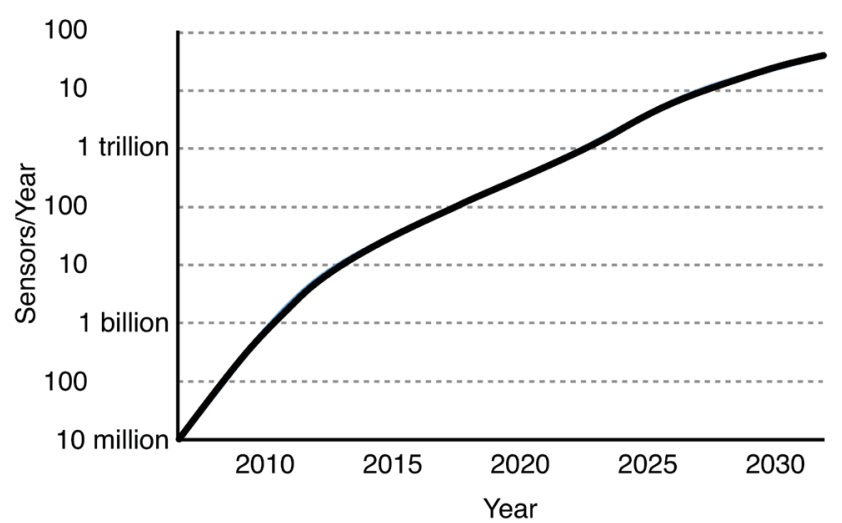

Fig. 2. Prediction of future annual number of sensors used.

activities and detecting infants' excretion in a diaper). Moreover, the price of the entire system has been markedly reduced.

To realize and develop a society with the above concepts-IoT and Trillion Sensor Universethe development of a platform for RF sensor systems is demanded. This platform is assumed to be a one-chip (i.e., monolithic) device that has various types of versatile MEMS sensors (e.g., those for humidity, atmospheric temperature and pressure, sunlight, sound, acceleration, geomagnetism, angular velocity), simple signal processing and RF functions, and an autonomous power unit. Although required sensors are different depending on purposes, sufficiently various types of sensor are mounted onto the platform in advance. Unnecessary sensors do not have to be used. This enables the use of common hardware and cost reduction by mass production, which is an advantage of MEMS technology. From the viewpoint of business, hybrid integration (system in package, where sensor and processor chips are integrated into a package) is currently considered to be advantageous. ${ }^{(5)}$ However, I think that the monolithic integration of the entire system (system on a chip) will be a big breakthrough for the Trillion Sensor Universe.

\section{Centenarian Sensor System}

\subsection{Lifetime of MEMS}

The targets of IoT application include intelligent transport systems, medical and health-related systems, security systems, home energy management systems (HEMSs), and factory energy management systems (FEMSs). In addition, structural health monitoring for buildings, bridges, tunnels, and railroads has attracted attention. Because structures such as bridges are generally designed to have a useful life of at least 20-100 years, sensor systems for monitoring such structures should have a higher durability performance, that is, they have to work for more than 100 years (I named them as centenarian sensor systems). If sensor systems can be designed from this viewpoint, the realization of maintenance-free structural health monitoring will become a real possibility. As the factors for determining the lifetime of MEMS sensors, not only the lifetime of the sensor mechanism itself but also that affected by assembling and packaging should be considered. Chemical sensors using enzymes essentially have a short lifetime (because the lifetime 
of enzymes is, for example, several hours to dozens of days), and cannot be used for centenarian sensor systems (although there is an eccentric idea of designing a pseudoliving system "artificial organisms" that produces enzymes by itself). On the other hand, for physical sensors with movable sections such as acceleration sensors and gyros, the fatigue of silicon should be considered but its mechanism has been still unclear. Initially, silicon was considered to be free from metal fatigue. However, it is found that fatigue in silicon progresses to some extent by the movement of internal defects even in an inert gas. Few MEMS manufacturers clearly present the indices for fatigue and failure, such as the mean time to failure (MTTF) and the failure in time (FIT). Analog Devices Inc., however, reveals that the FIT in the wafer process for micromachines is 0.1 at a humidity of $60 \%$ and a temperature of $55{ }^{\circ} \mathrm{C},{ }^{(8)}$ showing the FIT lower than that for general integrated circuits (for example, 2.64 for $0.13 \mu \mathrm{m}$ CMOS). SiTime states that the SiT15xx series MEMS oscillator exhibits an FIT of 2. ${ }^{(9)}$ Thus, general MEMS devices are considered to have a sufficient lifetime comparable to other electronic components and it may be sufficiently possible to achieve an FIT of $\sim 100-1000$ (corresponding to a MTTF of $\geq 100$ years) over the entire system (note that care should be taken to avoid the use of low-lifetime components in the system). The remaining problem lies in the power sources that generally use chemical reaction, that is, batteries.

\subsection{Energy harvesting technology}

Power supply is not a problem if the system is constantly accessible to commercial power sources. However, many of the future IoT devices will not satisfy this condition (because they are demanded to be used as mobile devices or placed at arbitrary positions). To meet such demands, systems should be equipped with an internal power source. Currently used standard power sources are batteries such as $\mathrm{Li}$-ion batteries, which use chemical reaction and are never durable for 100 years. Moreover, the need for regular maintenance such as charging is incongruent with the concept of centenarian sensor systems. As a solution to this problem, the energy harvesting technology for generating power from environmental energy becomes effective. Available environmental energies include sunlight, vibration, temperature change (temperature difference), and radio waves. Table 1 shows a summary of their energy densities. ${ }^{(10)}$ When placed outside, devices can obtain energy from sunlight several orders of magnitude higher than from other energy sources. However, sunlight is unavailable for devices used inside tunnels and clothes and those embedded into structural materials for buildings. Therefore, attempts to harvest energy from vibration, heat, and radio waves have been promoted. Here, I briefly introduce vibration-powered devices. ${ }^{(11,12)}$ According to the principle of power generation, vibration-powered devices are classified into three types:

Table 1

Power densities of energy sources.

\begin{tabular}{lccc}
\hline Source & Class & Power $/ \mathrm{cm}^{2}$ & Power $/ \mathrm{cm}^{3}$ \\
\hline Light & Outdoor & $100 \mathrm{~mW} / \mathrm{cm}^{2}$ & - \\
& Indoor & $100 \mu \mathrm{W} / \mathrm{cm}^{2}$ & - \\
Vibration & Low freq. $\sim \mathrm{Hz}$ & - & $\sim 4 \mu \mathrm{W} / \mathrm{cm}^{3}$ \\
& High freq. $\sim \mathrm{kHz}$ & - & $\sim 800 \mu \mathrm{W} / \mathrm{cm}^{3}$ \\
Thermal & Human & $\sim 60 \mu \mathrm{W} / \mathrm{cm}^{2}$ & - \\
& Industrial & $\sim 10 \mathrm{~mW} / \mathrm{cm}^{2}$ & - \\
RF & WiFi & $\sim 1 \mathrm{nW} / \mathrm{cm}^{2}$ & - \\
Acoustic & Noise: $75 \mathrm{~dB}$ & - & $\sim 3 \mathrm{nW} / \mathrm{cm}^{3}$ \\
\hline
\end{tabular}


piezoelectric, electromagnetic, and electrostatic types. For piezoelectric-type vibration-powered devices, a structure that can be distorted by vibration is formed and equipped with a piezoelectric element (that generates electric charges when distorted) at the distortable section to obtain power. Electromagnetic-type vibration-powered devices use electromagnetic induction caused by the relative movement of magnets and coils. For electrostatic-type vibration-powered devices, power is generated following the principle in which electric charges are induced in the fixed electrode as a result of the relative movement of the nonvolatile charges on the movable electrode. Here, an electrostatic-type energy harvester using an electret (a dielectric that semipermanently holds electric charges) is introduced. Figure 3(a) shows our prototype vibration-powered energy harvester. ${ }^{(13)}$ This consists of three layers: the topmost first layer is an electrode for collecting electric charges, the second layer comprises a silicon structure with an electret having the nonvolatile electric charges corresponding to 100-200 V on the surface and a spring that supports the structure, and the third layer is a glass substrate for protecting a movable mass. Electric charges are accumulated on the electret in a blind pattern. As shown in Fig. 3(b), when vibration (displacement of the mass) is given to the device, the electret moves to induce positive and negative charges in the electrode, thus generating power. Figure 4 shows photographs of a device on a 4-inch wafer and a chip obtained after dicing. Although details are not explained here, the mass of this device vibrates with the maximum amplitude of $200 \mu \mathrm{m}$ at a resonance frequency of $352 \mathrm{~Hz}$ and an acceleration of $8 \mathrm{G}$ and generates a power of $33 \mu \mathrm{W}$, which is converted to the volume energy density of $\sim 150 \mu \mathrm{W} / \mathrm{cm}^{3}$. The power generation can be further increased by decreasing the distance between the electrode and the electret and increasing the amount of electric charges. Most of such types of vibration-powered device has a secondary vibration system comprising a mass and a spring. For such types, the power generation markedly decreases at vibration frequencies other than the resonance frequency. Therefore, the use of a nonlinear system for generating large power over a wide frequency range has been intensively attempted. ${ }^{(14)}$

(a)

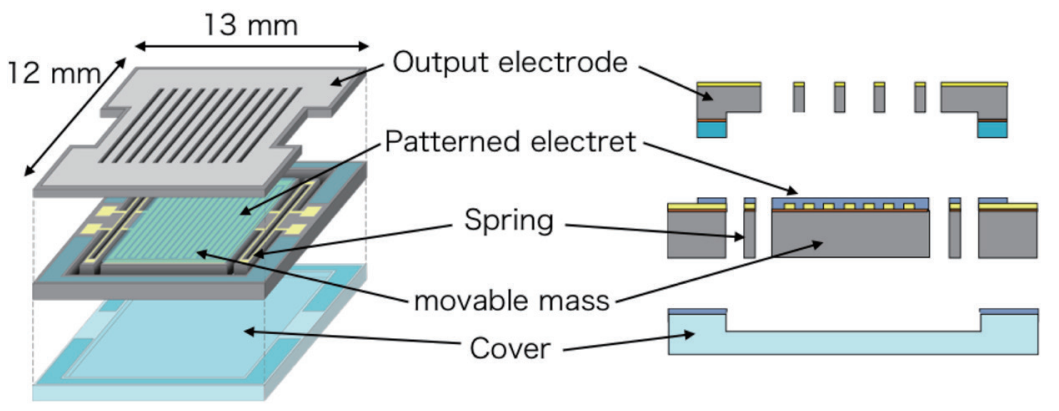

(b)

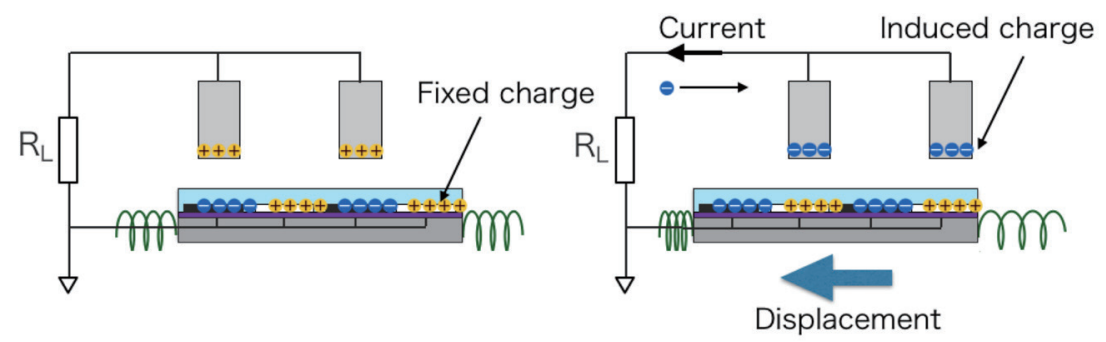

Fig. 3. (Color online) Example structure of electrostatic-type power harvester. 

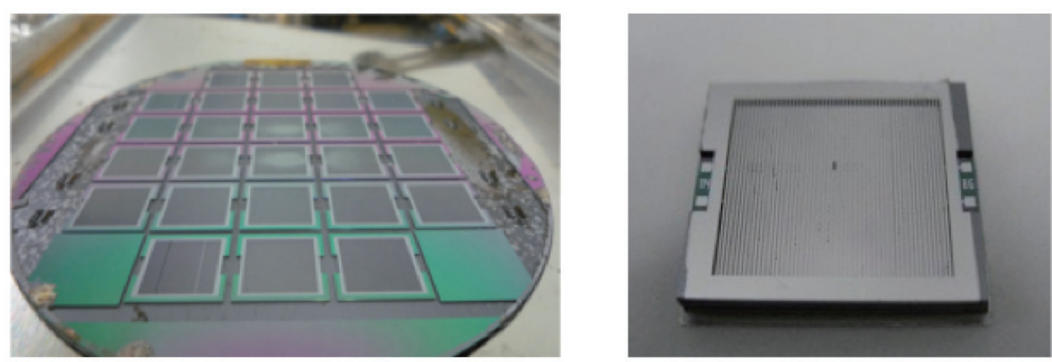

$12 \times 13 \times 1.5 \mathrm{~mm}^{3}$

Fig. 4. (Color online) Photograph of electrostatic-type power harvester.

The power generated by current energy harvesters is still small. However, some products have already been commercially available and attracted attention. Various ideas have also been proposed continuously. In addition, the power consumption of sensor systems has yearly decreased. I expect that completely autonomous wireless sensor systems will be realized in the near future.

\section{Incorporation of Actuators into Sensor System}

In a previous report, ${ }^{(6)}$ I described, "Assuming that a human is an automaton, it is an excellent automaton. ... This excellent automaton deserves attention as a model for sensor networks that will advance in various directions in the future". Sensors of living organisms including humans have an important characteristic. That is, ALL sensors are equipped with actuators, as shown in Fig. 5. Actuators have various functions, such as the expansion of the dynamic range during sensing (e.g., pupil, crystalline lens), maintenance and cleaning (eyelid, lacrimal apparatus, saliva, tongue), protection (eyelid, lip), assistance of sensing functions (olfactory sense with airflow by respiration; tactile sense for detecting shear stress by moving fingers on an object = acquiring data on surface state), and the addition of new functions (e.g., specifying the direction of a sound source by turning one's neck). Currently, most of the commercially available sensing devices have only sensing functions. I think it important to add actuator's functions to future higher-order sensing systems. In particular, the centenarian sensors mentioned in the previous section may be required to have some actuators for cleaning and maintaining the sensors and self-diagnosis. The above statement has not been authorized at academic societies but I introduce this as my opinion.

\section{Infrastructure of Network}

\subsection{Power consumption}

Today, the infrastructure related to the Internet, including cloud infrastructure, has been growing. Figure 6 shows the Internet protocol (IP) traffic expected in the future. ${ }^{(15)}$ Compared with the growth rate of fixed wire connection, the increase in traffic from mobile devices is significant (more than fourfold over five years). To accommodate this increase in traffic, the power consumed by routers used to relay data on the network and servers (cloud) used to store data is continuously increasing. It is estimated that the power consumed by the routers alone will exceed the total power generation in Japan in the 2020s if the traffic will increase at a rate of $40 \%$ per year. From this 


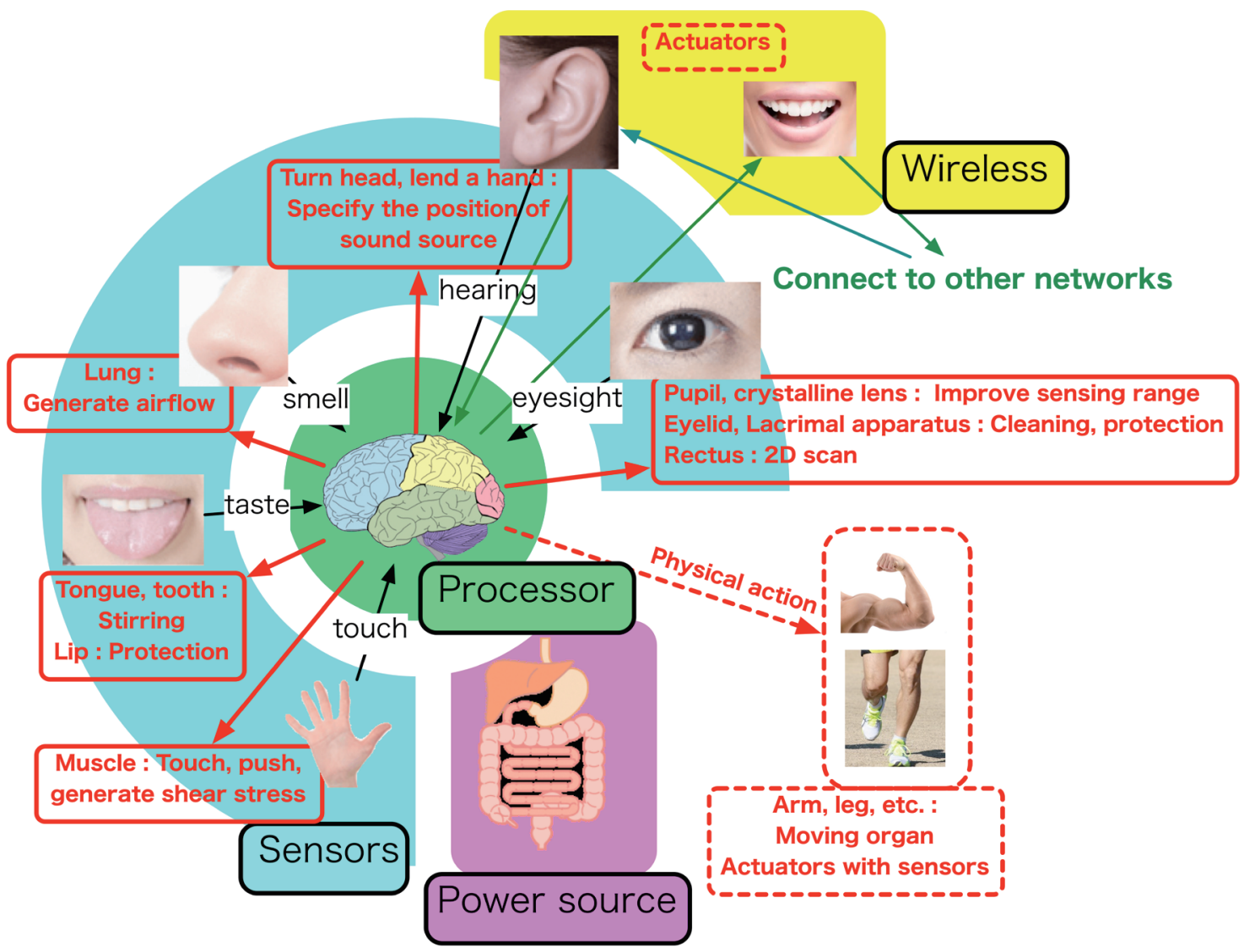

Fig. 5. (Color online) Conceptual diagram of human as automaton and its sensors and associated actuators.

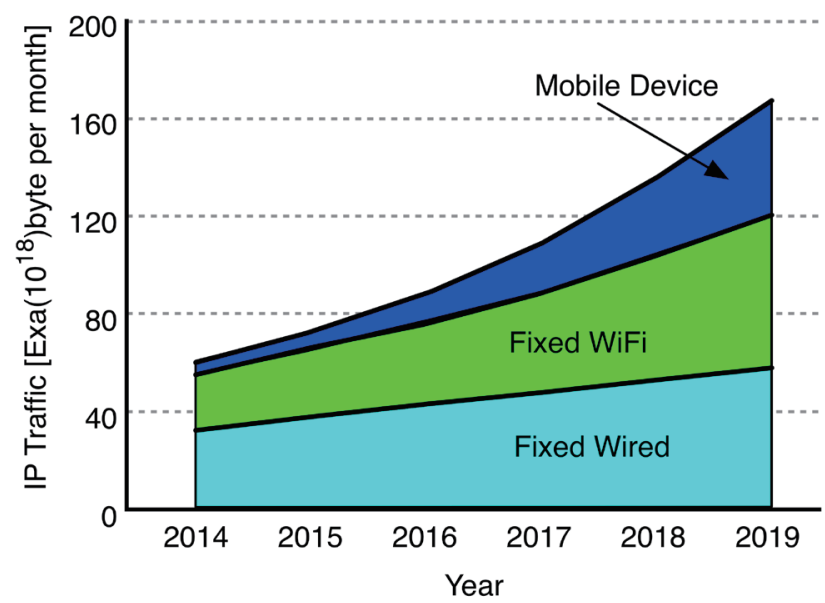

Fig. 6. (Color online) Prediction of future total traffic on the Internet.

viewpoint, the amount of data transmitted from sensor nodes (IoT devices) to the network should be minimized as much as possible and the increase in traffic of less informative or low-value data should be avoided. To this end, a certain level of data processing or semantic extraction in sensor 
nodes is indispensable. In addition, local processing without sending out the data to the network should be carried out if local feedback alone is acceptable. For example, a marked reduction in traffic is possible if the following algorithm is implemented in human activity monitoring systems, instead of sending out all raw data to the network to be analyzed at the cloud side: the criteria of health condition are determined first and the system issues an alert sound or haptic signals to the user only when the health condition of the user is judged to deteriorate (e.g., onset of heatstroke) if the condition continues. Moreover, if appropriate action is not taken despite the alert sound or haptic signals, the system sends out alert data to the network. With the Trillion Sensor Universe, the reduction in the power consumption not only by sensors but also via the network should be considered.

\subsection{Secrecy of data and big data processing}

In the future IoT society, large amounts of personal information, such as biological signals and behavioral history, and information that has not been disclosed to the public will be stored in servers via networks. Researchers in the field of sensors and materials should recognize the handling of such information, in particular, secrecy of data. Moreover, data automatically obtained by sensors will be so-called big data. In these days, attention is being paid to big data processing such as artificial intelligence (AI) and deep learning. Data that have been obtained by various sensors and seem to be unrelated to each other may be combined by big data processing and sorted out to obtain new meaning and understanding. For example, clarifying the relationships between the global environment and diseases, health and safety, infection route/speed and regional characteristics, and weather and traffic jam will contribute to the development of a safe, secure, and comfortable society. Sensor and IoT devices are being rapidly developed, but there are still many tasks to analyze big data obtained by these devices and understand the meaning of the data. I hope that big data are analyzed with the greatest care for the secrecy and safety of data.

\section{Summary}

Through the five sessions of this series with the theme of Sensors in Network, I have explained the developmental history, current status, and future prospects of sensors and their applications and technologies by focusing on sensors that will be used in the future IoT and Trillion Sensor Universe society. Although the concept of the sensor network itself was proposed previously, sensors, processors, RF technologies, power sources, and network infrastructures have made progress and enabled everyone to construct sensor networks easily. As mobile phones were evolved into new devices (beyond telephones) by becoming equipped with a camera function, sensor networks still have a room to receive novel ideas and greatly change to new ones. I expect the future progress of sensor networks.

\section{References}

1 K. Ashton: That 'Internet of Things' Thing, RFID Journal, 22 June, 2009.

2 The Internet of Things explained: evolutions, possibilities and realities, i-Scoop: http://www.i-scoop.eu/ internet-of-things/ (accessed Nov. 2016).

3 TSensors Summits for Trillion Sensor Roadmap, Stanford Univ., 23-25 Oct., 2013: www.tsensorssummit.org/ Resources/TSensors\%20Roadmap\%20v1.pdf (accessed Nov. 2016). 
4 K. Maenaka: Sens. Mater. 28 (2016) 745.

5 K. Maenaka: Sens. Mater. 28 (2016) 927.

6 K. Maenaka: Sens. Mater. 28 (2016) 1077.

7 K. Maenaka: Sens. Mater. 28 (2016) 1149.

8 Calculated by Analog Devices Website: http://www.analog.com/en/about-adi/quality-reliability/reliabilitydata/wafer-fabrication-data.html (accessed Nov. 2016).

9 S. Taranovich: SiTime Enters Smartphone Market with First MEMS Oscillator, EDN Network, 11 Apr., 2013.

10 D. Lehmhus and S. Bosse eds.: Structural Materials and Processes in Transportation (Wiley, May 2013) Chap. 17.

11 P. D. Mitcheson, E. M. Yeatman, G. K. Rao, A. S. Holmes, and T. C. Green: Proc. IEEE 96 (2008) 1457.

12 T. J. Kazmierski and S. Beeby eds.: Energy Harvesting Systems (Springer-Verlag, New York, 2011).

13 N. Miwatani, T. Fujita, K. Minami, K. Kanda, and K. Maenaka: Electrostatic Energy Harvester Using Doubledensity Counter Electrode for Wireless Sensor Systems, The 33rd Sensor Sym. on Sensors, Micromachines and Applied Systems, 25pm2-C-4, Hirado, Japan 2016 (in Japanese).

14 S. Priya and D. J. Inman eds.: Energy Harvesting Technologies (Springer-Verlag, US, 2009).

15 Cisco Visual Networking Index: Global Mobile Data Traffic Forecast Update, 2015-2020 White Paper, 1 Feb 2016: http://www.cisco.com/c/en/us/solutions/collateral/service-provider/visual-networking-index-vni/ mobile-white-paper-c11-520862.html (accessed Nov. 2016).

\section{About the Author}

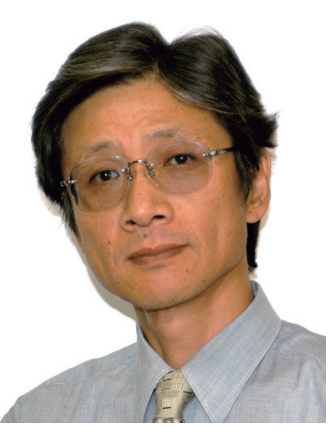

Kazusuke Maenaka received his B.E., M.E., and Ph.D. degrees from Toyohashi University of Technology, Japan, in 1982, 1984, and 1990, respectively. Since 1993, he has been with the Department of Electronics of Himeji Institute of Technology. With the unification of universities in the Hyogo prefecture in April 2004, he joined University of Hyogo, where he is presently a professor. From 2008 to 2013, he was the project leader of the Maenaka Human-Sensing Fusion Project supported by the Japan Science and Technology Agency. His research interests include MEMS devices and technology, especially silicon mechanical sensors and their integration. 